\title{
Energy concentration and Sommerfeld condition for Helmholtz equation with variable index at infinity
}

\author{
Benoit Perthame $\left({ }^{*}\right)$ and Luis Vega $\left({ }^{* *}\right)$ \\ $\left(^{*}\right)$ Ecole Normale Supérieure, DMA, UMR8553 \\ 45, rue d'Ulm 75230 Paris, France \\ email: benoit.perthame@ens.fr \\ $\left.{ }^{* *}\right)$ Universidad del Pais Vasco, Apdo. 644 \\ 48080 Bilbao, Spain \\ email: mtpvegol@lg.ehu.es
}

\begin{abstract}
We consider the Helmholtz equation with a variable index of refraction $n(x)$, which is not necessarily constant at infinity but can have an angular dependency like $n(x) \rightarrow n_{\infty}(x /|x|)$ as $|x| \rightarrow \infty$. Under some appropriate assumptions on this convergence and on $n_{\infty}$ we prove that the Sommerfeld condition at infinity still holds true under the explicit form

$$
\int_{\mathbb{R}^{d}}\left|\nabla u-i n_{\infty}^{1 / 2} u \frac{x}{|x|}\right|^{2} \frac{d x}{|x|}<+\infty .
$$

It is a very striking and unexpected feature that the index $n_{\infty}$ appears in this formula and not the gradient of the phase as established by Saito in [25] and broadly used numerically. This apparent contradiction is clarified by the existence of some extra estimates on the energy decay. In particular we prove that

$$
\int_{\mathbb{R}^{d}}\left|\nabla_{\omega} n_{\infty}\left(\frac{x}{|x|}\right)\right|^{2} \frac{|u|^{2}}{|x|} d x<+\infty .
$$

In fact our main contribution is to show that this can be interpreted as a concentration of the energy along the critical lines of $n_{\infty}$. In other words, the Sommerfeld condition hides the main physical effect arising for a variable $n$ at infinity; energy concentration on lines rather than dispersion in all directions.
\end{abstract}

\section{Introduction}

We consider the Helmholtz equation with a variable index of refraction $n(x)$, with a slow, and only radial decay to a constant $n_{\infty}(x /|x|)$ at infinity

$$
i \varepsilon u_{\varepsilon}+\Delta u_{\varepsilon}+n(x) u_{\varepsilon}=-f(x), \quad \varepsilon>0 .
$$

Our main interest is the so called limiting absorption principle (i.e. to study the limit when $\varepsilon>0$ approaches to 0 in (1.1)) and the validity of the Sommerfeld radiation condition at infinity. One of the main results in this paper is to prove that

$$
\int_{\mathbb{R}^{d}}\left|\nabla u(x)-i n_{\infty}^{1 / 2}\left(\frac{x}{|x|}\right) u(x) \frac{x}{|x|}\right|^{2} \frac{d x}{|x|}<+\infty .
$$


A direct consequence of this condition is the more classical setting

$$
\liminf \int_{|x|=r}\left|\nabla u(x)-i n_{\infty}^{1 / 2}\left(\frac{x}{|x|}\right) u(x) \frac{x}{|x|}\right|^{2} d \sigma(x) \rightarrow 0, \quad \text { as } \quad r \rightarrow \infty,
$$

where $d \sigma$ denotes the Lebesgue measure on the sphere. It is a very striking and unexpected feature that the term

$$
n_{\infty}^{1 / 2}\left(\frac{x}{|x|}\right) \frac{x}{|x|}
$$

appears in this formula instead of $\nabla \varphi(x)$ with $\varphi(x)$ the solution to the corresponding eikonal equation

$$
|\nabla \varphi|^{2}=n
$$

as established by Saito in [25]. This phenomenon, as well as the proof of (1.3), can be explained by the existence of some new energy estimate that we state later on and which is, in some sense, the main result of this paper. It explains that the Sommerfeld radiation condition hides the main physical effect of a variable $n$ at infinity; energy is not dispersed in all directions but concentrated on those given by the critical points of $\nabla n\left(\frac{x}{|x|}\right)$. It would be interesting to prove that only local maxima of $n$ can carry energy.

These estimates use in a strong way the inequality obtained in [21] for the tangential part of the gradient of the solution of (1.1). In order to recall that result we need some notation. Firstly we define, for $j \in \mathbb{Z}$, the annulus $C(j)$ by

$$
C(j)=\left\{x \in \mathbb{R}^{d} \text { s.t. } 2^{j} \leq|x| \leq 2^{j+1}\right\} .
$$

Then we set

$$
\begin{gathered}
\|\| u \|_{R_{0}}^{2}:=\sup _{R>R_{0}} \frac{1}{R} \int_{B(R)}|u|^{2} d x \\
N_{R_{0}}(f):=\sum_{j>J}\left[2^{j+1} \int_{C(j)}|f|^{2} d x\right]^{1 / 2}+\left[R_{0} \int_{B_{R_{0}}}|f|^{2} d x\right]^{1 / 2},
\end{gathered}
$$

with $J$ defined by $2^{J} \leq R_{0}<2^{J+1}$, and we drop the index $R_{0}$ if $R_{0}=0$. We also denote the radial and tangential derivatives by

$$
\frac{\partial}{\partial r} u(x):=\frac{x}{|x|} \cdot \nabla u(x), \quad \nabla_{\tau} u(x)=\frac{\partial}{\partial \tau} u(x):=\nabla u(x)-\frac{x}{|x|} \frac{\partial}{\partial r} u
$$

and for a function $n(\omega) \in C^{1}\left(S^{d-1}\right)$, we shall consider

$$
\nabla_{\omega} n(\omega)=\frac{\partial}{\partial \omega} n(\omega):=|x| \frac{\partial}{\partial \tau} n\left(\frac{x}{|x|}\right), \quad \omega=\frac{x}{|x|} .
$$

Let us consider the following assumptions:

$$
n=n_{1}+n_{2} \quad \text { with } n_{2} \in L^{\infty}, \quad n>0
$$




$$
\begin{aligned}
& \left\|\left(n_{1}\right)^{1 / 2} u\right\|_{2}<\left(1-c_{0}\right)\|\nabla u\|_{2} \text { for all smooth functions } u \text { and some } c_{0}>0 \\
& \qquad 2 \sum_{j \in \mathbb{Z}} \sup _{C(j)} \frac{(x \cdot \nabla n(x))_{-}}{n(x)}:=\beta<1
\end{aligned}
$$

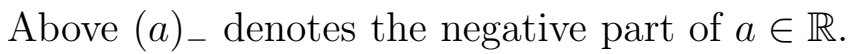

In [21] we prove the following result.

Theorem 1.1 We assume one of the following two conditions:

(i) $d \geq 3$, (1.7) - (1.9) and $R_{0}=0$;

(ii) $d=2$, (1.7)-(1.9), $n>n_{0}>0$ and $R_{0}=n_{0}^{-1 / 2}$;

Then the solution to the Helmholtz equation (1.1) satisfies,

$$
\begin{aligned}
M^{2}:=\|\nabla u\|_{R_{0}}^{2}+\left\|n^{\frac{1}{2}} u\right\|_{R_{0}}^{2} & +\int_{|x| \geq R_{0}} \frac{\left|\nabla_{\tau} u\right|^{2}}{|x|} d x \\
& \leq C\left(\varepsilon+\left\|n_{2}\right\|_{\infty}\right) N_{R_{0}}\left(\frac{f}{n^{1 / 2}}\right)^{2} .
\end{aligned}
$$

The homogeneity of the above estimate makes it compatible with the high frequencies (replace $n$ by $\mu^{2} n$ ). Our main interest in [21] was to obtain estimates with the right scaling. In particular we were able to recover the well known inequality for $u$ of Agmon and Hörmander in [3] in the constant coefficient case. Similar results but not scaling invariant were obtained in [15] and [28. The scaling plays a fundamental role in the applications to nonlinear Schrödinger equations ([16]) and in the high frequency limit for Helmholtz equations ([6], [7]). In this paper we get an explicit Sommerfeld radiation condition for solutions obtained from the limiting absorption principle. As we have already said the estimate of the tangential component of the gradient

$$
\int_{|x| \geq R_{0}} \frac{\left|\nabla_{\tau} u(x)\right|^{2}}{|x|} d x<\infty
$$

given in (1.10) turns out to be fundamental. In order to get it we need the smallness assumption given in (1.9). We do not know if it is necessary or not. However the condition in (1.9) is necessary and can not be relaxed to a Coulomb type of decay, even if smallness is added. This is proved in the appendix using as counterexamples a family of wave guides for which the estimate of the tangential derivative is false.

In order to prove the new energy estimate, we need to impose some extra assumptions on $n$. They are the following ones:

$$
\text { there exists } \quad n_{\infty}\left(\frac{x}{|x|}\right) \in C^{3}\left(S^{d-1}\right), \quad n_{\infty}\left(\frac{x}{|x|}\right) \geq n_{0}>0,
$$

and

$$
\left|n(x)-n_{\infty}\left(\frac{x}{|x|}\right)\right| \leq n_{\infty}\left(\frac{x}{|x|}\right) \frac{\Gamma}{|x|}, \quad \Gamma>0 \quad n>0
$$


In fact and as we shall prove in section 2 this last assumption (1.13) can be largely relaxed if for example $n-n_{\infty}$ is radial -see (2.2), (2.3) below.

We may now state our basic new estimate (already announced in [23]). Its interest relies of course on the bounds stated in Theorem 1.1.

Theorem 1.2 For dimensions $d \geq 2$, we assume (1.9), (1.12) and (1.13) and use the notation of Theorem 1.1. Then the solution to the Helmholtz equation (1.1) satisfies, for $R \geq R_{0}$ and $R$ large enough

$$
\bar{M}:=\int_{|x| \geq R}\left|\nabla_{\omega} n_{\infty}\left(\frac{x}{|x|}\right)\right|^{2} \frac{|u(x)|^{2}}{|x|} d x \leq C\left[\left(\varepsilon+\|n\|_{\infty}\right) N_{R_{0}}\left(\frac{f}{n^{1 / 2}}\right) M+M^{2}\right]
$$

for some constant $C$ independent of $\epsilon$.

We would like to point out the sharpness of this inequality. It says that the points where $\left|\nabla_{\omega} n_{\infty}\left(\frac{x}{|x|}\right)\right|$ vanishes on the sphere are the concentration directions for the energy $|u|^{2}$. Indeed, we can derive from the Sommerfeld condition below the following proposition. The proof can be found at the end of section 3 .

Proposition 1.3 With the assumptions of Theorem 1.4 below, we have

$$
\lim _{\mathrm{R} \rightarrow \infty} \frac{1}{R} \int_{|x| \leq R} n_{\infty}^{1 / 2}\left(\frac{x}{|x|}\right)|u(x)|^{2} d x=-\mathcal{I} m \int_{R^{d}} f(x) \bar{u}(x) d x .
$$

Therefore if $u$ vanishes in a neighborhood of the critical points of $n_{\infty}$ we get the bound

$$
\int_{|x| \geq R} \frac{|u(x)|^{2}}{|x|} d x<\infty
$$

and deduce that $\mathcal{I} m \int_{\mathbb{R}^{d}} f(x) \bar{u}(x) d x=0$. From the Sommerfeld radiation condition and in the constant coefficient case this leads ([12, p. 242) to a restrictive condition on the Fourier transform of $f$, namely $\widehat{f}(\xi)=0$ on the sphere $|\xi|=n^{1 / 2}$. It would be interesting to explore which should be the conclusion in our setting. A natural hypothesis is to substitute $\widehat{f}(\xi)$ by the generalized Fourier transform defined by S. Agmon, J. Cruz-Sampedro and I. Herbst in [2] which depends upon the construction of solutions to the associated eikonal equation. The role played by the critical points of $n_{\infty}$ was already pointed out by I. Herbst in [11. There are two related estimates that have been deduced by the same method, the case of stationary kinetic equations (the high frequency limit of Helmholtz equations), see [22], and the case of two layers, see [10], where the gradient of $n_{\infty}$ gives a surface Dirac mass.

Our next purpose is to give an explicit Sommerfeld radiation condition for the solution obtained by the limiting absorption principle. Our result complements that of Saito in [25]. Therefore we shall assume

$$
n(x)=\lambda+p(x), \quad \lambda>0,
$$

with $p$ a bounded real function which belongs to $C^{2}\left(\mathbb{R}^{n} \backslash\{0\}\right)$ and such that

$$
\left|\partial_{x}^{\alpha} p(x)\right| \leq c|x|^{-|\alpha|}, \quad|\alpha| \leq 2 .
$$


Theorem 1.4 For dimensions $d \geq 2$, assume (1.9), (1.17) and (1.16). Then for $\lambda$ large enough compared to $\|p\|_{\mathcal{C}^{2}(|x|>1)}$, there exists a unique solution to the Helmholtz equation with $\varepsilon=0, M<\infty$, and which satisfies for any $a>1$,

$$
\int_{\mathbb{R}^{d}}\left|\nabla u(x)-i n^{1 / 2}(x) u(x) \frac{x}{|x|}\right|^{2} \frac{d x}{1+|x|} \leq C_{a} \int_{\mathbb{R}^{d}}|f(x)|^{2}(1+|x|)^{a} .
$$

Moreover if there are $n_{\infty}, \Gamma>0$ and $\delta>0$ such that

$$
\left|n(x)-n_{\infty}\left(\frac{x}{|x|}\right)\right| \leq n(x) \frac{\Gamma}{|x|^{\delta}} \quad \text { for }|x| \text { large enough },
$$

then from (1.10) and (1.18) we get

$$
\int_{\mathbb{R}^{d}}\left|\nabla u(x)-i n_{\infty}^{1 / 2}(x) u(x) \frac{x}{|x|}\right|^{2} \frac{d x}{1+|x|} \leq C_{a} \int_{\mathbb{R}^{d}}|f(x)|^{2}(1+|x|)^{a} .
$$

Let us compare the above theorem with previously known results. There is a very extensive literature on the limiting absorption principle, see for example [8], 9], 11, [17, [5], 15, [27] and references there in. The situation for the Sommerfeld radiation condition is different. When $n=\lambda+V(x)$ and $V$ is a short range potential the question was settled by Ikebe and Saito in [14]. Mochizuku and Uchiyama study in [20] large range potentials with mild radial oscillations at infinity like $V(x) \sim \sin (\ln |x|)$. Hörmander in [13, chapter XXX, characterizes the incoming/outgoing solutions obtained from the limiting absorption principle by some asymptotic behavior, but in his case $n_{\infty}=\lambda=$ constant. More general long range potentials were considered by Saito in [25]. Although in this latter work perturbations of first order terms ("magnetic potentials") are also considered let us fix the attention in the conditions for $V$. Saito writes $V=p+Q$ where $Q$ is a short range perturbation, while $p$ satisfies (1.17). Then he proves a Sommerfeld radiation condition for $\lambda$ large enough given by $\nabla u \pm i \sqrt{\lambda}(\nabla \varphi) u$, where $\varphi$ is an appropriate solution for $|x|>R_{0}, R_{0}$ large enough, of the associated eikonal equation 1

$$
|\nabla \varphi|^{2}=1+\frac{p(x)}{\lambda}
$$

Therefore one cannot expect that in general the vector $\nabla \varphi$ points at the direction $x /|x|$. An illustrative example is to consider

$$
p(x)=-\frac{x_{1}}{|x|} .
$$

In this case and for $\lambda$ large enough, see Remark 1.3 in [25], $\varphi(x)=a(\lambda)|x|-b(\lambda) x_{1}$ with $a(\lambda)=1 / 2\left[(1+1 / \lambda)^{1 / 2}+(1-1 / \lambda)^{1 / 2}\right]$ and $b(\lambda)=1 / 2\left[(1+1 / \lambda)^{1 / 2}-(1-1 / \lambda)^{1 / 2}\right]$. This boundary condition differs from ours in all points except when $\nabla n=0$ (here $n=\lambda+p$ ). Then the apparent contradiction is clarified thanks to the estimate (1.14) which applies for this example.

\footnotetext{
${ }^{1}$ The existence of this solution was established later on by Barles 4 .
} 
Notice however that the assumptions for Theorem 1.2 and for Theorem 1.4 are different and not comparable. In the particular case $n=n_{\infty}$ and regular, no smallness assumption is needed in Theorem 1.2 because (1.9) is trivially fulfilled, while $\lambda$ has to be large to construct the solution of the eikonal equation, which is a fundamental step in order to prove Theorem 1.4. On the other hand Saito's assumption (1.17) with $n=\lambda+p$ doesn't imply the existence of the limit $n_{\infty}$.

In the proof of Theorem 1.4 is crucial the estimate of the tangential part of the gradient given in (1.10) to conclude that for $\varphi$ given in (1.21)

$$
\int\left|\nabla_{\tau} \varphi(x) u(x)\right|^{2} \frac{d x}{1+|x|}<+\infty
$$

This is an energy estimate in itself which says that $u$ concentrates along the critical points of $\nabla_{\tau} \varphi$. In section 3 we prove that under some conditions these critical points coincide with those of $\nabla_{\tau} n_{\infty}$ establishing a relation between the energy estimate given in Theorem 1.2 and that in (1.22). Notice however that for the proof of Theorem 1.2 we do not need the existence of a solution to the eikonal equation (1.21) which well could not exist.

The paper is organized as follows. In section 2 we prove Theorem 1.2. Section 3 is devoted to the study of the eikonal equation following [4. In particular we give some properties of the corresponding solution. The proof of Theorem 1.4 is given in section 4. In Appendix 1 we gather some elementary identities and in Appendix 2 we give the example of the family of wave guides mentioned above.

Acknowledgments. We want to thank T. Hoffmann-Ostenhoff, and G. Barles for enlightening conversations. We also thank E. Fouassier for pointing out the shortcomings of a previous version.

\section{Proof of Theorem 1.2}

We are going to prove a more general version. In fact we will consider two different ways of measuring $\left|n-n_{\infty}\right|$. First recall the assumption (1.13)

$$
\left|n(x)-n_{\infty}\left(\frac{x}{|x|}\right)\right| \leq n(x) \frac{\Gamma}{|x|}, \quad \Gamma>0 .
$$

We can instead assume the alternative conditions

$$
\begin{gathered}
\left|n(x)-n_{\infty}\left(\frac{x}{|x|}\right)\right| \leq n \frac{\Gamma}{|x|^{\delta}} \quad \text { for }|x|>R_{0}, \quad \Gamma>0, \quad \delta>0, \quad \text { and } R_{0}>1 \\
\left\{\begin{array}{l}
\text { there exists } \tilde{\beta}<1, \delta>0 \text { and } \bar{\Gamma}>0 \text { such that } \\
\left(|x| \nabla_{\tau}\left(n-n_{\infty}\right) \cdot \frac{\partial n_{\infty}}{\partial \omega}\right)_{-} \leq \tilde{\beta}\left|\frac{\partial n_{\infty}}{\partial \omega}\right|^{2}+n(x) \frac{\bar{\Gamma}}{|x|^{\delta}} .
\end{array}\right.
\end{gathered}
$$


In particular if $n-n_{\infty}$ is radial (2.2) is sufficient. Also note that from (1.12) and either (2.1) or (2.2) we conclude that

$$
|n| \leq C \quad n \geq \frac{n_{0}}{2} \quad \text { for }|x| \text { large enough. }
$$

Let us start recalling the fundamental ingredients of the proof of Theorem 1.1 given in [21. In fact in that paper we discarded the estimate for the tangential derivative because it was of not use. However, and as we already said in the introduction, (1.11) turns out to be fundamental in the proof of Theorem 1.2 and Theorem 1.4 .

Consider for $R>0$ the special functions $\Psi, \phi$ given by

$$
\begin{aligned}
& \nabla \Psi=\left\{\begin{array}{ccc}
\frac{x}{R} & \text { for } & |x| \leq R, \\
\frac{x}{|x|} & \text { for } & |x| \geq R,
\end{array}\right. \\
& \phi=\left\{\begin{array}{lll}
\frac{1}{2 R} & \text { for } & |x| \leq R, \\
0 & \text { for } & |x| \geq R .
\end{array}\right.
\end{aligned}
$$

We will also need the following formulas which hold in the distributional sense

$$
\begin{gathered}
D_{i j}^{2} \Psi=\left\{\begin{array}{l}
\frac{\delta_{i j}}{R} \quad \text { for }|x| \leq R, \\
\left(\delta_{i j}|x|^{2}-x_{i} x_{j}\right) /|x|^{3} \text { for }|x| \geq R,
\end{array}\right. \\
\Delta \Psi=\left\{\begin{array}{c}
\frac{d}{R} \text { for }|x| \leq R, \\
(d-1) /|x| \text { for } \quad|x| \geq R .
\end{array}\right.
\end{gathered}
$$

A simple calculation gives for $d>2$

$$
\frac{1}{4} \int_{\mathbb{R}^{\mathrm{d}}} v \Delta(2 \phi-\Delta \Psi) \geq \frac{d-1}{4 R^{2}} \int_{S(R)} v d \sigma_{R} .
$$

As it is well known $\Delta^{2} \Psi$ is positive for $d=2$, and has to be treated in a different way. We refer to section 5 in [21] for the corresponding modifications.

Adding the identity (5.1) to (5.3) with the above choices of $\Psi$ and $\phi$ we get

$$
\begin{gathered}
\frac{1}{2 R} \int_{B(R)}|\nabla u|^{2}+\int_{|x|>R}\left(|\nabla u|^{2}-\left|\frac{x}{|x|} \cdot \nabla u\right|^{2}\right) /|x| \\
+\frac{d-1}{4 R^{2}} \int_{S(R)}|u|^{2} d \sigma_{R}+\frac{1}{2 R} \int_{B(R)}(n(x)+x \cdot \nabla n(x))|u|^{2} \\
+\frac{1}{2} \int_{|x|>R} \frac{x}{|x|} \cdot \nabla n(x)|u|^{2} \leq \\
-\mathcal{R} e\left[\frac{1}{R} \int_{B(R)} f(x)\left(x \cdot \nabla \bar{u}+\frac{d-1}{2} \bar{u}\right)+\int_{|x|>R} f(x)\left(\frac{x}{|x|} \cdot \nabla \bar{u}+\frac{d-1}{2|x|} \bar{u}\right)\right]
\end{gathered}
$$




$$
-\varepsilon \mathcal{I} m\left[\frac{1}{R} \int_{B(R)} x \cdot \nabla u \bar{u}+\int_{|x|>R} \frac{x}{|x|} \cdot \nabla u \bar{u}\right] .
$$

We shall estimate separately the various terms in the right-hand side of the above inequality. We begin by the four terms containing $f$. The pairs containing $f$ and $\nabla u$ is easy to bound by the right-hand side of (1.10) because the two norms defined in (1.4) and (1.5) are one dual of the other one (see also the next argument). So we concentrate on the other two terms we have for $\delta>0$

$$
\begin{aligned}
\int_{\mathbb{R}^{\mathrm{d}}}|f(x)| \frac{|u|}{|x|} & \leq \sum_{j \in \mathbb{Z}}\left(2^{-j} \int_{C(j)} \frac{|u|^{2}}{|x|^{2}}\right)^{1 / 2}\left(2^{j} \int_{C(j)}|f(x)|^{2}\right)^{1 / 2} \\
\leq & \left(\sup _{R} \frac{1}{R^{2}} \int_{S(R)}|u|^{2} d \sigma_{R}\right)^{1 / 2} \sum_{j \in \mathbb{Z}}\left(2^{j} \int_{C(j)}|f(x)|^{2}\right)^{1 / 2} \\
& \leq \delta \sup _{R} \frac{1}{R^{2}} \int_{S(R)}|u|^{2} d \sigma_{R}+C_{\delta} N(f)^{2} .
\end{aligned}
$$

We consider now the terms on $n$. We have,

$$
\begin{gathered}
\frac{1}{R} \int_{\mathbb{R}^{\mathrm{d}}}(\nabla \Psi \cdot \nabla n)_{-}|u|^{2} \leq \sum_{j \in \mathbb{Z}} \int_{C(j)} n|u|^{2} \frac{(x \cdot \nabla n)_{-}}{|x| n} \\
\leq\left(\sup _{R} \frac{1}{R} \int_{B(R)} n|u|^{2}\right) \sum_{j \in \mathbb{Z}} \sup _{C(j)} 2^{j+1} \frac{(x \cdot \nabla n)_{-}}{|x| n} \leq \beta_{1} \sup _{R} \frac{1}{R} \int_{B(R)} n|u|^{2} .
\end{gathered}
$$

Finally we have to bound the terms involving $\varepsilon$. From the Helmholtz equation one deduces

$$
\begin{gathered}
\varepsilon \int_{\mathbb{R}^{\mathrm{d}}}|u|^{2} \leq \int_{\mathbb{R}^{\mathrm{d}}}|f \bar{u}|, \\
\int_{\mathbb{R}^{\mathrm{d}}}|\nabla u|^{2} \leq \int_{\mathbb{R}^{\mathrm{d}}} n|u|^{2}+\int_{\mathbb{R}^{\mathrm{d}}}|f \bar{u}| .
\end{gathered}
$$

With the assumptions (1.7) and (1.8) we obtain

$$
\int_{\mathbb{R}^{\mathrm{d}}}|\nabla u|^{2} \leq C\left(\int_{\mathbb{R}^{\mathrm{d}}} n_{2}|u|^{2}+\int_{\mathbb{R}^{\mathrm{d}}}|f \bar{u}|\right) .
$$

Using Cauchy-Schwarz inequality and the above inequalities we get

$$
\begin{gathered}
\varepsilon \int_{\mathbb{R}^{\mathrm{d}}}|\nabla u||u| \leq C \varepsilon^{1 / 2}\left(\int_{\mathbb{R}^{\mathrm{d}}} n_{2}|u|^{2}+\int_{\mathbb{R}^{\mathrm{d}}}|f \bar{u}|\right)^{1 / 2}\left(\int_{\mathbb{R}^{\mathrm{d}}}|f \bar{u}|\right)^{1 / 2} \\
\leq C \varepsilon^{1 / 2} \int_{\mathbb{R}^{\mathrm{d}}}|f \bar{u}|+C\left(\varepsilon \sup \left|n_{2}\right| \int_{\mathbb{R}^{\mathrm{d}}}|f \bar{u}| \int_{\mathbb{R}^{\mathrm{d}}}|u|^{2}\right)^{1 / 2} \\
\leq C\left(\varepsilon+\sup \left|n_{2}\right|\right)^{1 / 2} \int_{\mathbb{R}^{\mathrm{d}}}|f \bar{u}|
\end{gathered}
$$




$$
\begin{gathered}
\leq C\left(\varepsilon+\sup \left|n_{2}\right|\right)^{1 / 2}\left\|\left|n^{1 / 2} u \|\right| N\left(\frac{f}{n^{1 / 2}}\right)\right. \\
\leq\left.\delta\left\|n^{1 / 2} u\right\|\right|^{2}+C_{\delta}\left(\varepsilon+\sup \left|n_{2}\right|\right)\left(N\left(\frac{f}{n^{1 / 2}}\right)\right)^{2} .
\end{gathered}
$$

Then plug (2.11)-(2.13) into (2.9) and take the supremum in $R$. Then we get (1.10) and in particular (1.11).

Let us prove now Theorem 1.2. The proof consists in using the basic equality (5.3) with a test function that depends on the behavior of $n(x)$ at infinity. We choose for $R \geq R_{0}$ such that (2.4) holds and define

$$
\Psi_{q}(x)=q\left(\frac{|x|}{R}\right) n_{\infty}\left(\frac{x}{|x|}\right)
$$

for some non-decreasing smooth function $q(r)=0$ for $r \leq 1$ and $q(r)=r$ for $r \geq 2$.

With this choice, we will show that the only new information (compared to Theorem 1.1) in (5.3), is given by the term

$$
\int_{R^{d}} \nabla n(x) \cdot \nabla \Psi_{q}(x)|u(x)|^{2} d x
$$

Then we will take care of the other terms using (1.10).

As a first step we consider (2.14). We simplify the notation using $q=q\left(\frac{|x|}{R}\right)$. Then we get

$$
\begin{gathered}
\int_{R^{d}} \nabla n(x) \cdot \nabla \Psi_{q}(x)|u(x)|^{2} d x=\int_{R^{d}} q\left|\frac{\partial}{\partial \omega} n_{\infty}(\omega)\right|^{2} \frac{|u(x)|^{2}}{|x|^{2}} d x \\
+\int_{R^{d}} \frac{\partial}{\partial r} n(x) \frac{q^{\prime}}{R} n_{\infty}(\omega)|u(x)|^{2} d x+\int_{R^{d}} q|x| \nabla_{\tau}\left(n(x)-n_{\infty}\left(\frac{x}{|x|}\right)\right) \frac{\partial}{\partial \omega} n_{\infty}(\omega) \frac{|u(x)|^{2}}{|x|^{2}} d x .
\end{gathered}
$$

The first term on the right-hand side gives the control we look for. As for the second term we just have to consider the negative part of $\frac{\partial}{\partial r} n(x)$. Then we proceed as in (2.12) to get the lower bound

$$
-\frac{C}{R} \beta\left\|n_{\infty}\right\|_{L^{\infty}}\left\|n^{1 / 2} u\right\|_{R_{0}}^{2} .
$$

Let us consider first condition (2.1). Then the last term in (2.15) is bounded below as follows. After integration by parts, it is also given by

$$
\begin{gathered}
-\mathcal{R} e \int_{R^{d}} \frac{q}{|x|^{2}}\left(n(x)-n_{\infty}\left(\frac{x}{|x|}\right)\right)\left(D_{\omega}^{2} n_{\infty}|u(x)|^{2}+2 \frac{\partial}{\partial \omega} n_{\infty}(\omega)|x| \nabla_{\tau} u(x) \bar{u}\right) d x \\
\geq-\Gamma\left[\left\|n_{\infty}\right\|_{C^{2}} \int_{\mathbb{R}^{d}} q n(x) \frac{|u(x)|^{2}}{|x|^{3}} d x+2\|n\|_{L^{\infty}} \int_{\mathbb{R}^{d}} \frac{q}{|x|^{2}}\left|\frac{\partial}{\partial \omega} n_{\infty}(\omega)\right|\left|\nabla_{\tau} u(x)\right||u| d x\right] \\
\geq-\left.\frac{C}{R^{2}}\left\|n_{\infty}\right\|{C^{2}}^{2}\left|\left\|n^{1 / 2} u\right\|_{R}^{2}-\frac{1}{2} \int_{\mathbb{R}^{d}} q\right| \frac{\partial}{\partial \omega} n_{\infty}(\omega)\right|^{2} \frac{|u(x)|^{2}}{|x|^{2}} d x
\end{gathered}
$$




$$
-\frac{C}{R}\|n\|_{L^{\infty}}^{2} \int_{|x| \geq R} \frac{\left|\nabla_{\tau} u(x)\right|^{2}}{|x|} d x .
$$

Let us assume now (2.2), then the last term of (2.15) is bounded below by

$$
-\tilde{\beta} \int_{\mathbb{R}^{d}} q\left|\frac{\partial}{\partial \omega} n_{\infty}(\omega)\right|^{2} \frac{|u(x)|^{2}}{|x|^{2}} d x-\frac{C}{R^{\delta}}\left\|n^{1 / 2} u\right\|_{R_{0}}^{2} .
$$

As a conclusion of this first step we have obtained

$$
\begin{gathered}
\int_{\mathbb{R}^{d}} q\left(\frac{|x|}{R}\right)\left|\frac{\partial}{\partial \omega} n_{\infty}(\omega)\right|^{2} \frac{|u(x)|^{2}}{|x|^{2}} d x \\
\leq C_{1} \int_{\mathbb{R}^{d}} \nabla n(x) \cdot \nabla \Psi_{q}(x)|u(x)|^{2} d x+C_{2} M^{2} .
\end{gathered}
$$

The second step is to provide a control on all the remaining terms thanks to the basic equality (5.3).

We have

$$
\begin{gathered}
\nabla \bar{u}(x) \cdot D^{2} \Psi_{q}(x) \cdot \nabla u(x)=\frac{q^{\prime}}{R|x|} n_{\infty}(\omega)\left|\nabla_{\tau} u\right|^{2}+\frac{q^{\prime \prime}}{R^{2}} n_{\infty}(\omega)\left|\frac{\partial}{\partial r} u\right|^{2} \\
+2\left(\frac{q^{\prime}}{R|x|}-\frac{q}{|x|^{2}}\right) \mathcal{R} e\left[\frac{\partial}{\partial r} \bar{u} \frac{\partial}{\partial \omega} n_{\infty}(\omega) \cdot \nabla_{\tau} u\right]+\frac{q}{|x|^{2}} \nabla_{\tau} \bar{u} \cdot D_{\omega}^{2} n_{\infty}(\omega) \cdot \nabla_{\tau} u .
\end{gathered}
$$

And because the terms $\left(\frac{q^{\prime}}{|x|}-\frac{q}{|x|^{2}}\right)$ and $q^{\prime \prime}$ are supported in the ball $\{|x| \leq R\}$, we see that from (1.10) all the terms in the corresponding integral are bounded by $C\left\|n_{\infty}\right\|_{C^{2}} M / R$.

Next, we consider the term

$$
\begin{gathered}
\int_{\mathbb{R}^{d}} \Delta^{2} \Psi_{q}(x)|u(x)|^{2}=-2 \mathcal{R} e \int_{\mathbb{R}^{d}} \nabla \Delta \Psi_{q} \cdot \nabla u \bar{u} \\
\leq 2 \int_{\mathbb{R}^{d}} \frac{\left|\nabla \Delta \Psi_{q}\right|}{n^{1 / 2}}|\nabla u|\left|n^{1 / 2} u\right| .
\end{gathered}
$$

But we have

$$
\left|\nabla \Delta \Psi_{q}(x)\right| \leq C\left\|n_{\infty}\right\|_{C^{3}}\left[\frac{q}{|x|^{3}}+\frac{q^{\prime}}{R|x|^{2}}\right] \leq c \frac{\|n\|_{\infty}}{|x|^{2}} .
$$

Therefore from (1.10) we get that (2.18) is bounded. by

$$
C\left\|\frac{n_{\infty}}{n_{0}^{1 / 2}}\right\|_{C^{3}} M / R
$$

The right-hand side terms containing $f$ can be treated as in (2.11) and are respectively upper bounded by

$$
C\left\|n_{\infty}\right\|_{C^{1}} N_{R_{0}}(f)\|\nabla u\|_{R_{0}}
$$


and by

$$
C\left\|\frac{n_{\infty}}{n_{0}^{1 / 2}}\right\|_{C^{2}} N_{R_{0}}(f)\left\|n^{1 / 2} u\right\|_{R_{0}} .
$$

The last term to be bounded is

$$
\varepsilon \mathcal{I} m \int_{\mathbb{R}^{d}} \nabla \Psi_{q}(x) \cdot \nabla \bar{u}(x) u(x) .
$$

But this is done as in (2.13). The proof of Theorem 1.2 is over.

\section{The eikonal equation}

In order to determine the phase arising in the Sommerfeld radiation condition, we need to solve the eikonal equation

$$
\left\{\begin{array}{l}
|\nabla \varphi|^{2}=\frac{1}{\lambda} n(x)=1+\frac{1}{\lambda} p(x), \quad x \in \mathbb{R}^{d}, \quad \lambda>0 \\
\varphi(0)=0, \quad \nabla \varphi(x) /|x| \text { bounded }
\end{array}\right.
$$

where we assume that $\lambda>0$ is large enough and that $p \in C^{2}\left(\mathbb{R}^{d}\right)$ and satisfies

$$
\left|D^{\alpha} p(x)\right| \leq C(1+|x|)^{-\alpha}, \quad \text { for }|\alpha| \leq 2 .
$$

In order to take into account the linear growth at infinity (which is essential for uniqueness), the unique viscosity solution $\varphi$ to (3.1) is better described in terms of the bounded function $g(x)$ defined as

$$
\varphi(x)=|x| g(x) .
$$

This change of unknown yields the following Hamilton-Jacobi equation for $g$ :

$$
\left\{\begin{array}{l}
|g|^{2}+2 r g \partial_{r} g+|x|^{2}|\nabla g|^{2}=1+\frac{1}{\lambda} p(x), \quad x \in \mathbb{R}^{d} \backslash\{0\}, \\
g(0)=\sqrt{\frac{n(0)}{\lambda}}
\end{array}\right.
$$

From this H.-J. equation, one can derive properties of the phase $\varphi$, which we state now.

\subsection{Statements of the results}

Let us recall the results of Barles given in [4].

Theorem 3.1 (Barles, [4]) Under assumption (3.2), there exists a unique solution to (3.3). Moreover it satisfies the following estimates, for $\lambda$ large enough and $x \neq 0$,

$$
\begin{gathered}
\sqrt{1-\min p / \lambda} \leq g(x) \leq \sqrt{1+\max p / \lambda} \\
|x||D g(x)| \leq C / \lambda, \quad|x|^{2}\left|D^{2} g(x)\right| \leq C / \lambda, \quad|x|^{3}\left|D^{3} g(x)\right| \leq C / \lambda .
\end{gathered}
$$


In the proof of Theorem 1.4 we shall derive the estimate

$$
\int\left|\nabla_{\tau} \varphi u\right|^{2} \frac{d x}{1+|x|}<+\infty,
$$

which is similar to our estimate (1.14) in Theorem 1.2. Therefore it is a natural question to know which is the relation between the two inequalities (1.14) and (3.5). In fact we shall see that under some extra assumptions on the index $n$, both inequalities are equivalent. Indeed, assume

$$
\left.\left|\partial_{r} p(x)\right| \leq C r^{-1-\delta}, \quad \text { for some } \delta \in\right] 0,1[,
$$

with $r=|x|$ and $\partial_{r}=\frac{x}{|x|} \cdot \nabla$ the radial derivative. Firstly, as a consequence of this assumption, $n(x)$ admits a radial limit

$$
n\left(r \frac{x}{|x|}\right) \rightarrow n_{\infty}\left(\frac{x}{|x|}\right) \quad \text { as } r \rightarrow \infty
$$

and in fact the decay rate in our previous assumption (1.19) also follows from (3.6). Then, we have

Theorem 3.2 Under assumptions (3.2) and (3.6), the solution to (3.3) satisfies for $\lambda$ large enough and $x \neq 0$ the estimate,

$$
r^{1+\delta}\left|\partial_{r} g(x)\right| \leq C / \lambda,
$$

and, as in (3.7), $g\left(r \frac{x}{|x|}\right) \rightarrow g_{\infty}\left(\frac{x}{|x|}\right)$ as $r \rightarrow \infty$, a smooth solution to the equation

$$
g_{\infty}(\omega)^{2}+\left|\nabla_{\omega} g_{\infty}(\omega)\right|^{2}=n_{\infty}(\omega), \quad \omega \in \mathbb{S}^{d-1} .
$$

Moreover

$$
\left|\nabla_{\tau} \varphi\right|=\left|\nabla_{\omega} g_{\infty}(\omega)\right|+O\left(r^{-\delta}\right)
$$

Remark. The solution to the limiting equation (3.9) enjoys a specific property. From (3.4) we get that $\left(g_{\infty} I+D_{\omega}^{2} g_{\infty}\right)$ is invertible and after differentiation in (3.9) we obtain

$$
0<c_{1}\left|\nabla_{\omega} g_{\infty}\right| \leq\left|\nabla_{\omega} n_{\infty}\right| \leq c_{2}\left|\nabla_{\omega} g_{\infty}\right|
$$

Therefore the critical points (and thus the extrema) of $n_{\infty}$ and $g_{\infty}$ coincide.

The derivation of Theorem 3.2 can be seen either from the representation through the method of characteristics or from the more modern PDE point of view. We give both proofs for the sake of completeness. 


\subsection{Proof of Theorem 3.2 based on bicharacteristics}

We decompose the proof in two steps. We first recall the definition of the characteristics and how they give a representation formula for the solution to the eikonal equation (3.1), then we come to the proof of the estimates in Theorem 3.2

First step. Characteristics. The bicharacteristics are classically defined as the solutions to the differential system, parameterized by some $q \in \mathbb{S}^{d}$,

$$
\begin{cases}\dot{X}(t ; q)=2 P(t ; q), & X(t=0 ; q)=0, \\ \dot{P}(t ; q)=\frac{1}{\lambda} \nabla n(X(t ; q)), & P(t=0 ; q)=\frac{1}{\lambda^{1 / 2}} q \sqrt{n(0)},|q|^{2}=1, \\ \dot{\Phi}(t ; q)=\frac{2}{\lambda} n(X(t ; q)), & \Phi(t=0 ; q)=0 .\end{cases}
$$

We note for later purpose that

$$
\frac{d}{d t}\left[|P|^{2}-\frac{1}{\lambda} n(X)\right]=0, \text { therefore }|P(t ; q)|^{2}=\frac{1}{\lambda} n(X(t ; q)) \forall t \in \mathbb{R} .
$$

Assume first that we can define a unique diffeomorphism (this involves smallness assumptions)

$$
\left.x \in \mathbb{R}^{d} \backslash\{0\} \rightarrow(t, q) \in\right] 0, \infty\left[\times \mathbb{S}^{d-1}, \quad x=X(t ; q) .\right.
$$

Then, we recall the standard representation formula for the solution $\varphi(x)$ to (3.1) (see [18] for details and complements).

Lemma 3.3 For all $t>0$ and $q \in \mathbb{S}^{d-1}$ such that condition (3.12) holds, we have

$$
P(t ; q)=\nabla \varphi(X(t ; q)), \quad \varphi(X(t ; q))=\Phi(t ; q),
$$

for some smooth solution $\varphi(x)$ to (3.1).

Proof. Because both the H.-J. solution and the differential system (3.11) are stable by smooth perturbations of $n$, we can assume that $n$ is constant in a neighborhood of the origin. Then, we divide the proof in three steps.

(i) Then, close to $x=0$ the solution to the H.-J. equation is given $\varphi(x)=\frac{1}{\lambda^{1 / 2}} \sqrt{n(0)}|x|$ and the bicharacteristics are

$$
X(t ; q)=2 t q \sqrt{n(0)}, P=q \sqrt{n(0)}=\nabla \varphi(x), \Phi=2 n(0) t=\sqrt{n(0)}|X|=\varphi(x) .
$$

Hence the result holds true.

(ii) We prove that $P(t ; q)$ is a gradient, i.e., $\left(\frac{\partial P}{\partial x}\right)$ is a symmetric matrix. Indeed, from (i), it is true for $t \approx 0$ and it remains to see that for all times $\frac{d}{d t}\left(\frac{\partial P}{\partial x}\right)$ is a symmetric matrix. But we 
can compute

$$
\begin{aligned}
\frac{d}{d t}\left(\frac{\partial P}{\partial x}\right) & =\frac{d}{d t}\left[\left(\frac{\partial P}{\partial(t, q)}\right)\left(\frac{\partial X}{\partial(t, q)}\right)^{-1}\right] \\
& =\left(\frac{\partial \dot{P}}{\partial(t, q)}\right)\left(\frac{\partial X}{\partial(t, q)}\right)^{-1}+\left(\frac{\partial P}{\partial(t, q)}\right) \frac{d}{d t}\left(\frac{\partial X}{\partial(t, q)}\right)^{-1} \\
& =\frac{1}{\lambda} D^{2} n(X(t ; q))\left(\frac{\partial X}{\partial(t, q)}\right)\left(\frac{\partial X}{\partial(t, q)}\right)^{-1}-\left(\frac{\partial P}{\partial(t, q)}\right)\left(\frac{\partial X}{\partial(t, q)}\right)^{-1}\left(\frac{\partial \dot{X}}{\partial(t, q)}\right)\left(\frac{\partial X}{\partial(t, q)}\right)^{-1} \\
& =\frac{1}{\lambda} D^{2} n(X(t ; q))-2\left(\frac{\partial P}{\partial(t, q)}\right)\left(\frac{\partial X}{\partial(t, q)}\right)^{-1}\left(\frac{\partial P}{\partial(t, q)}\right)\left(\frac{\partial X}{\partial(t, q)}\right)^{-1} \\
& =\frac{1}{\lambda} D^{2} n(X(t ; q))-2\left(\frac{\partial P}{\partial x}\right)\left(\frac{\partial P}{\partial x}\right) \\
& =2 P \cdot\left(\frac{\partial^{2} P}{\partial x^{2}}\right)
\end{aligned}
$$

because $|P|^{2}=\frac{1}{\lambda} n$ implies $\left(\frac{\partial P}{\partial x}\right)\left(\frac{\partial P}{\partial x}\right)+P \cdot\left(\frac{\partial^{2} P}{\partial x^{2}}\right)=\frac{1}{2 \lambda}\left(\frac{\partial^{2} n}{\partial x^{2}}\right)$. We have obtained indeed that $\frac{d}{d t}\left(\frac{\partial P}{\partial x}\right)$ is a symmetric matrix and our claim is proved.

(iii) From step (ii), we can write $P(t ; q)=\nabla \varphi(X(t ; q))$ for some function $\varphi$. And since $|P|^{2}=$ $\frac{1}{\lambda} n$, we obtain that $\varphi$ solves the H.-J. equation. It remains to identify $\Phi$ and $\varphi$. To do so, we write

$$
\frac{d}{d t} \varphi(X(t ; q))=\nabla \varphi(X(t ; q)) \frac{d}{d t} X(t ; q)=2|P(t ; q)|^{2}=\frac{2}{\lambda} n(X(t ; q)) .
$$

This means that $\varphi(X(t ; q))=\Phi(t ; q)$ since the identity holds at $t=0$ and the two functions have the same derivatives. This completes the proof of Lemma 3.3 .

The reciprocal to Lemma 3.3 is also true. If the H.-J. solution is smooth, then we can derive that the bicharacteristic system satisfies the invertibility condition. As before, for simplicity we assume that $n$ is constant in a neighborhood of the origin.

Lemma 3.4 Assume that the solution $\varphi$ to H.-J. equation is smooth and satisfies $x \cdot \nabla \varphi(x) \geq$ $c|x|$ and consider the autonomous differential equation

$$
\frac{d}{d t} X(t ; q)=\nabla \varphi(X(t ; q)), \quad X(0 ; q)=0, \quad X(t ; q) \approx n^{1 / 2}(0) q t(t \text { small }) .
$$

This gives the solution to (3.11) with the formulas of Lemma 3.3.

Proof. We prove successively that:

(i) it is the solution to the bicharacteristic system;

(ii) $\left(t>0, q \in \mathbb{S}^{d-1}\right) \mapsto X(t ; q) \in \mathbb{R}^{d} \backslash\{0\}$ is one-to-one;

(iii) it is a diffeomorphism.

The point (i) follows from the computation in the Lemma 3.3 and we do not reproduce them again. 
The point (ii) can be proved as follows.

(a) into : if $X\left(t_{0} ; q\right)=X\left(t_{0}+\tau ; q^{\prime}\right)$, by uniqueness of the system for backward time $X(t ; q)=$ $X\left(t+\tau ; q^{\prime}\right)$ for all $t$ and thus (considering what happens close to the origin) $\tau=0$ and $q=q^{\prime}$. (b) onto: take a point $y \neq 0$, and solve the equation (3.13) backward from say $t=0$ and call $Y(t, y)$ the solution. Because we know that $x \cdot \nabla \varphi(x) \geq c|x|$, we have

$$
\frac{d}{d t} \frac{|Y(t)|^{2}}{2} \geq c|Y(t)|
$$

Therefore it reaches $Y(\tau ; y)=0$ in finite time $(\tau<0)$. Now, the system (3.13) is autonomous. Therefore we can solve it from $t=0$, with $q_{0}=n(0)^{-1 / 2} \lim _{s \rightarrow 0} Y(\tau+s) / s$ and one has $y=X\left(-\tau ; q_{0}\right)$.

For point (iii), we just notice that the above construction, based on the Cauchy-Lipschitz theory, also provides $C^{1}$ regularity and $C^{1}$ regularity of the inverse.

These two lemmas show that, in the regime of assumption (3.2), with $\lambda$ large enough, the solution to the H.-J. equation is given by the bicharacteristics. We can use this fact to conclude the proof of Theorem 3.2 .

Second step. Estimates in Theorem 3.2. We divide the proof of these estimates in two steps. First an estimate on the rays is given, then we prove (3.8). Then, the second statement, (3.9), is an easy consequence obtained passing to the limit as $r \rightarrow \infty$.

(i) We have: $|X(t ; q)| / t=O(1)$. Indeed, we can compute

$$
\frac{d}{d t} \frac{|X|^{2}}{2}=X \cdot \dot{X}=2 X \cdot P
$$

and

$$
\frac{d}{d t} X \cdot P=2|P|^{2}+\frac{1}{\lambda} X \cdot \nabla n=\frac{2}{\lambda} n+\frac{1}{\lambda} X \cdot \nabla n .
$$

As a consequence of assumption (3.6), we have

$$
\frac{d^{2}}{d t^{2}} \frac{|X|^{2}}{4}=\frac{1}{\lambda} n+o(1) \text { for } X \text { large, }
$$

and thus the result (i) is proved.

(ii) We have: $|x|^{1+\delta} \partial_{r} g$ is bounded. Using the calculation of step (i), we have

$$
\begin{aligned}
\Phi(t ; q) & =\frac{2}{\lambda} \int_{0}^{t} n(X(s ; q)) d s=\int_{0}^{t}\left[\frac{d}{d t} X \cdot P-\frac{1}{\lambda} X \cdot \nabla n\right] d s \\
& =X \cdot P(t ; q)-F(t ; q)
\end{aligned}
$$

with

$$
F(t ; q)=\frac{1}{\lambda} \int_{0}^{t} X(s ; q) \cdot \nabla n(X(s ; q)) d s, \quad|F(x)| \leq C[1+|x|]^{1-\delta}
$$


In other words, for $x$ large we have

$$
\partial_{r} g=\frac{1}{|x|} \partial_{r} \varphi-\frac{\varphi}{|x|^{2}}=\frac{F(x)}{|x|^{2}}=O\left(\frac{1}{|x|^{1+\delta}}\right),
$$

and the claim (ii) is proved. This concludes the proof of (3.8) and thus the proof of Theorem 3.2 is complete.

\subsection{Proof of Theorem 3.2 based on H.-J. equations}

The proof is based on the equation for $\partial_{r} g$,

$$
2 g \partial_{r} g+r\left(\partial_{r} g\right)^{2}+r g \partial_{r r}^{2} g+r|\nabla g|^{2}+r^{2} \nabla g \cdot \nabla \partial_{r} g=\frac{1}{2 \lambda} \partial_{r} n .
$$

Upper bound. We consider the maximum point (if it is not attained, then perturbation methods, see [18, apply)

$$
\max \left[r^{1+\delta} \partial_{r} g(x)\right]=r_{0}^{1+\delta} \partial_{r} g\left(x_{0}\right),
$$

and at the point $x_{0}$, we have

$$
(1+\delta) \frac{x_{0}}{\left|x_{0}\right|} \partial_{r} g\left(x_{0}\right)+r \nabla \partial_{r} g\left(x_{0}\right)=0 .
$$

Inserting this in the equation on $\partial_{r} g$, we find

$$
2 g \partial_{r} g+r\left(\partial_{r} g\right)^{2}-(1+\delta) g \partial_{r} g+r|\nabla g|^{2}-(1+\delta) r \nabla g \cdot \frac{x}{|x|} \partial_{r} g\left(x_{0}\right)=\frac{1}{2 \lambda} \partial_{r} n
$$

Therefore we obtain

$$
(1-\delta)\left[g \partial_{r} g+r\left(\partial_{r} g\right)^{2}\right] \leq \frac{1}{2 \lambda} \partial_{r} n
$$

and thus we arrive at the upper bound

$$
\max \left[r^{1+\delta} \partial_{r} g(x)\right] \leq \frac{1}{2(1-\delta) \lambda} \max \left[r^{1+\delta} \partial_{r} n(x)\right] / \min g .
$$

Lower bound. With the same calculation as above, at the point $x_{1}$ where the minimum is attained

$$
\min \left[r^{1+\delta} \partial_{r} g(x)\right]=r_{1}^{1+\delta} \partial_{r} g\left(x_{1}\right)
$$

we have successively

$$
\begin{gathered}
(1-\delta) g \partial_{r} g-\delta r\left(\partial_{r} g\right)^{2}+r|\nabla g|^{2}=\frac{1}{2 \lambda} \partial_{r} n \\
(1-\delta) g \partial_{r} g-\delta r^{-(1+2 \delta)}\left(r^{1+\delta} \partial_{r} g\right)^{2}+\frac{1}{r}\left[\frac{n}{\lambda}-g^{2}-2 r g \partial_{r} g\right]=\frac{1}{2 \lambda} \partial_{r} n \\
(1+\delta) r^{1+\delta} g \partial_{r} g+\delta r^{-\delta}\left(r^{1+\delta} \partial_{r} g\right)^{2}=r^{\delta}\left[\frac{n}{\lambda}-g^{2}\right]-\frac{r^{1+\delta}}{2 \lambda} \partial_{r} n
\end{gathered}
$$

which gives the result. 


\section{Proof of Theorem 1.4 and of Proposition 1.3}

We will need the following uniqueness theorem.

Theorem 4.1 For dimensions $d \geq 2$, assume (1.7)-(1.9). If $u$ is a solution of $\Delta u+n u=0$ with $u$ and $\nabla u$ locally in $L^{2}$, and such that

$$
\liminf \int_{|x|=r}\left(|\nabla u|^{2}+|u|^{2}\right) d \sigma(x)=0, \quad \text { as } \quad r \rightarrow \infty
$$

then $u=0$.

Proof. Assume $R_{j}$ is a sequence going to infinity such that

$$
\lim _{\mathrm{j}} \int_{|x|=R_{j}}\left(|\nabla u|^{2}+|u|^{2}\right) d \sigma(x)=0, \quad \text { as } \quad j \rightarrow \infty .
$$

Consider $\Psi$ and $\phi$ as in (2.5) and (2.6) . Then fix $j$ and use the multiplier $\nabla \Psi \nabla \bar{u}+(1 / 2) \Delta \Psi \bar{u}+\phi \bar{u}$ in Helmholtz equation

$$
\Delta u+n u=0
$$

in the region $|x| \leq R_{j}$, and for $R \leq R_{j}$. Then we repeat the procedure given at the beginning of section 2 to prove Theorem 1.1. Note that just the multiplier which involves $\Psi$ will create boundary terms after the integration by parts. Therefore the basic identity (5.3) has to be modified. In this case we shall obtain

$$
\begin{aligned}
0= & \int_{B\left(0, R_{j}\right)} \nabla \bar{u}(x) D^{2} \Psi(x) \nabla u(x) d x \\
& -\frac{1}{4} \int_{B\left(0, R_{j}\right)}|u(x)|^{2} \Delta^{2} \Psi(x) d x+\frac{1}{2} \int_{B\left(0, R_{j}\right)}|u(x)|^{2}(\nabla n \cdot \nabla \Psi)(x) d x \\
& +\frac{1}{2} \int_{S_{R_{j}}} \partial_{r} \Psi(x)|\nabla u(x)|^{2} d \sigma(x)-\Re \int_{S_{R_{j}}} \partial_{r} u(x) \nabla \Psi(x) \cdot \nabla \bar{u}(x) d \sigma(x) \\
& +\frac{1}{4} \int_{S_{R_{j}}} \partial_{r} \Delta \Psi(x)|u(x)|^{2} d \sigma(x)-\frac{1}{4} \int_{S_{R_{j}}} \partial_{r}\left(|u(x)|^{2}\right) \Delta \Psi(x) d \sigma(x) \\
& +\frac{1}{2} \int_{S_{R_{j}}} \partial_{r} \Psi(x)|u(x)|^{2} n(x) d \sigma(x) .
\end{aligned}
$$


The inner terms are the same as in the proof of Theorem 1.1. Therefore adding those given by $\phi$ we shall obtain following the same argument given in section 2

$$
\begin{aligned}
\sup _{R \leq R_{j}} \frac{1}{R} \int_{B(0, R)} & \left(|\nabla u(x)|^{2} d x+n|u(x)|^{2}\right) d x \\
& \leq C \int_{S_{R_{j}}}\left|\partial_{r} \Psi(x)\right||\nabla u(x)|^{2} d \sigma(x)+\left|\Re \int_{S_{R_{j}}} \partial_{r} u(x) \nabla \Psi(x) \cdot \nabla \bar{u}(x) d \sigma(x)\right| \\
& +C \int_{S_{R_{j}}}\left|\partial_{r} \Delta \Psi(x)\right||u(x)|^{2} d \sigma(x)+C \int_{S_{R_{j}}}\left|\partial_{r}\left(|u(x)|^{2}\right)\right||\Delta \Psi(x)| d \sigma(x) \\
& +C \int_{S_{R_{j}}}\left|\partial_{r} \Psi(x)\right||u(x)|^{2} n(x) d \sigma(x) .
\end{aligned}
$$

Then taking the limit in $j$ the theorem follows from (4.2).

Proof of Theorem [1.4. Let us recall the estimate obtained by Saito in Theorem 1.6 of [25]. There is $a$, with $1<a \leq 2$ such that

$$
\int\left|\nabla u-i \lambda^{1 / 2} \nabla \varphi u\right|^{2} \frac{d x}{(1+|x|)^{2-a}} \leq c \int|f|^{2}(1+|x|)^{a} d x .
$$

In order to prove this inequality Saito needed the existence of $\varphi$, a solution of the eikonal equation for $|x|>R_{0}$ with $R_{0}$ large enough, which was established later on by Barles in [4], and that we gave in Theorem 3.1 of section 3 .

From (4.5) we get

$$
\int\left|\nabla u-i \lambda^{1 / 2} \nabla \varphi u\right|^{2} \frac{d x}{1+|x|} \leq c \int|f|^{2}(1+|x|)^{a} d x
$$

Hence looking at just the tangential parts of the above inequality and from (1.11) we get

$$
\int\left|\lambda^{1 / 2} \nabla_{\tau} \varphi u\right|^{2} \frac{d x}{1+|x|} \leq c \int|f|^{2}(1+|x|)^{a} d x
$$

From the eikonal equation we have that

$$
n-\lambda\left|\partial_{r} \varphi\right|^{2}=\left|\lambda^{1 / 2} \nabla_{\tau} \varphi\right|^{2}
$$

Recall that from the properties of $\nabla \varphi($ (3.4) $)$ we know that $\partial_{r} \varphi=g(x)+O(1 / \lambda)>0$. Then

$$
\left|\lambda^{1 / 2} \partial_{r} \varphi-n^{1 / 2}\right|=\frac{\left|\lambda^{1 / 2} \nabla_{\tau} \varphi\right|^{2}}{\left|\lambda^{1 / 2} \partial_{r} \varphi+n^{1 / 2}\right|} \leq c\left|\lambda^{1 / 2} \nabla_{\tau} \varphi\right|^{2}
$$

Also looking at the radial part in (4.6) we get

$$
\int\left|\partial_{r} u-i \lambda^{1 / 2} \partial_{r} \varphi u\right|^{2} \frac{d x}{1+|x|} \leq c \int|f|^{2}(1+|x|)^{a} d x .
$$


Finally from the above estimates we get

$$
\begin{gathered}
\int\left|\nabla u-i n^{1 / 2} \frac{x}{|x|} u\right|^{2} \frac{d x}{1+|x|}=\int\left(\left|\partial_{r} u-i n^{1 / 2} u\right|^{2}+\left|\nabla_{\tau} u\right|^{2}\right) \frac{d x}{1+|x|} \\
\leq c \int\left(\left|\partial_{r} u-i \lambda^{1 / 2} \partial_{r} \varphi u\right|^{2}+\left|\left(\lambda^{1 / 2} \partial_{r} \varphi-n^{1 / 2}\right) u\right|^{2}+\left|\nabla_{\tau} u\right|^{2}\right) \frac{d x}{1+|x|} \\
\leq c \int|f|^{2}(1+|x|)^{a} d x .
\end{gathered}
$$

Therefore we have proved (1.18).

Let us assume now that $\left|n-n_{\infty}\right|<c(1+|x|)^{-\delta}$. Then using (1.10) we conclude that

$$
\int\left|\nabla u-i n_{\infty}^{1 / 2} \frac{x}{|x|} u\right|^{2} \frac{d x}{1+|x|} \leq c \int|f|^{2}(1+|x|)^{a} d x
$$

which is (1.20).

Only the uniqueness remains to be proved. From

$$
\int\left|\nabla u-i n^{1 / 2} \frac{x}{|x|} u\right|^{2} \frac{d x}{1+|x|} \leq C
$$

we get that

$$
\liminf \int_{|x|=r}\left(\left|\nabla u-i n^{1 / 2} \frac{x}{|x|} u\right|^{2}\right) d \sigma(x)=0, \quad \text { as } \quad r \rightarrow \infty,
$$

Multiply in the equation $\Delta u+n u=0$ by $\bar{u}$ and integrate by parts in $|x| \leq R$. Taking the imaginary part one gets $\mathcal{I} m \int_{|x|=r} \bar{u} \partial_{r} u d \sigma(x)=0$. And therefore

$$
\mathcal{I} m \int_{|x|=R} \bar{u}\left(\partial_{r} u-i n^{1 / 2} u\right) d \sigma(x)=-\int_{|x|=R} n^{1 / 2}|u|^{2} d \sigma(x) .
$$

Then from (1.10), (4.7), (4.8), and that $n$ is bounded we get that $u$ satisfies (4.1). Therefore uniqueness follows from Theorem 4.1 .

Proof of Proposition 1.3. The argument is similar to the one to obtain (4.7). We multiply in the equation $\Delta u+n u=f$ by $\bar{u}$, integrate by parts in the ball of radius $R_{0}$ and take imaginary parts on both sides to obtain

$$
\mathcal{I} m \int_{|x|=R_{0}} \bar{u} f d \sigma(x)=\mathcal{I} m \int_{|x|=R_{0}} \bar{u}\left(\partial_{r} u-i n^{1 / 2} u\right) d \sigma(x)=-\int_{|x|=R_{0}} n^{1 / 2}|u|^{2} d \sigma(x) .
$$

Integrate in $R_{0}$ in the above expression for $0 \leq R_{0} \leq R$, and divide by $R_{0}$. Recall that from (1.16) $n \geq c_{0} \lambda$ for some $c_{0}>0$. Then the result follows from Sommerfeld radiation condition and (1.10). 


\section{Appendix}

A.1.- Basic identities. Our proof combines three basic identities that have been used throughout this paper and that we state here without proof (see [21] for a proof). For real valued functions $\Psi, \varphi, \psi \in \mathcal{S}\left(\mathbb{R}^{d}\right)$, we have

$$
\begin{gathered}
-\int_{\mathbb{R}^{d}} \varphi(x)|\nabla u(x)|^{2}+\frac{1}{2} \int_{\mathbb{R}^{d}} \Delta \varphi(x)|u(x)|^{2}+\int_{\mathbb{R}^{d}} \varphi(x) n(x)|u(x)|^{2} \\
=\mathcal{R} e \int_{\mathbb{R}^{d}} \varphi(x) \bar{u}(x) f(x) . \\
\varepsilon \int_{\mathbb{R}^{d}} \psi(x)|u(x)|^{2}-\mathcal{I} m \int_{\mathbb{R}^{d}} \nabla \psi(x) \cdot \nabla u(x) \bar{u}(x) \\
=\mathcal{I} m \int_{\mathbb{R}^{d}} f(x) \bar{u}(x) \psi(x) . \\
\int_{\mathbb{R}^{d}}\left[\nabla \bar{u}(x) \cdot D^{2} \Psi(x) \cdot \nabla u(x)-\frac{1}{4} \Delta^{2} \Psi(x)|u(x)|^{2}+\frac{1}{2} \nabla n(x) \cdot \nabla \Psi(x)|u(x)|^{2}\right]= \\
-\mathcal{R} e \int_{\mathbb{R}^{d}} f(x)\left(\nabla \Psi(x) \cdot \nabla \bar{u}(x)+\frac{1}{2} \Delta \Psi(x) \bar{u}(x)\right)-\varepsilon \mathcal{I} m \int_{\mathbb{R}^{d}} \nabla \Psi(x) \cdot \nabla \bar{u}(x) u(x) .
\end{gathered}
$$

A.2.- Some Examples. In this section we shall give examples of indices $n$ which satisfy

$$
\frac{(x \cdot \nabla n(x))_{-}}{n(x)}:=\tilde{\beta}<+\infty
$$

with $\tilde{\beta}$ as small as wanted and such that Theorem 1.1 does not hold true. In particular the estimate for the tangential derivative is false. Also we will exhibit the corresponding Sommerfeld radiation condition.

Condition (5.4) is weaker than (1.9) and appears naturally in the study of the absence of embedded eigenvalues in the continuous spectrum for the Schrödinger operator $\Delta+n$. Recall at this respect the well known example due to Von Neumann and Wigner of a potential which satisfies (5.4) for $\tilde{\beta}$ large enough and has an embedded eigenvalue, see [24] p. 233.

We give examples of wave guides which satisfy (5.4) but with a scaling which does not leave invariant that condition, and therefore there is no possible $\tilde{\beta} \operatorname{good}$ for all of them. For these examples condition (1.9) is not fulfilled either.

Define $Q$ as the unique positive solution with $Q( \pm \infty)=0$ of

$$
Q^{\prime \prime}+\left(Q^{2}-\frac{1}{2}\right) Q=0, \quad y \in \mathbb{R}
$$


That is to say $Q(y)=\operatorname{sech}(y / \sqrt{2})$. Also for $\lambda>0$ take $Q_{\lambda}(y)=Q(\lambda y)$, which solves

$$
Q_{\lambda}^{\prime \prime}+\lambda^{2}\left(Q_{\lambda}^{2}-\frac{1}{2}\right) Q_{\lambda}=0
$$

Set $\theta \in \mathcal{C}^{\infty}$ a bump function around the origin with $\theta(x)=0$ if $|x|<1$ and $\theta(x)=1$ if $|x|>2$. Then call

$$
u_{\lambda}^{\varepsilon}(x, y)=Q_{\lambda}(y) \theta(x) e^{i \sqrt{1+i \varepsilon}|x|},
$$

with $0<\varepsilon<1$ and $\mathcal{I} m \sqrt{1+i \varepsilon}>0$. Then $u_{\lambda}^{\varepsilon}$ solves

$$
\Delta u_{\lambda}^{\varepsilon}+\left(n_{\lambda}+i \varepsilon\right) u_{\lambda}^{\varepsilon}=f_{\varepsilon} \quad(x, y) \in \mathbb{R}^{2}
$$

with

$$
n_{\lambda}:=\tilde{n}_{\lambda}+1-\frac{1}{2} \lambda^{2} ; \quad \tilde{n}_{\lambda}:=\lambda^{2} Q^{2}(\lambda y)
$$

and

$$
f_{\varepsilon}(x, y)=\left(2 i \sqrt{1+i \varepsilon} \operatorname{sig}(x) \theta^{\prime}(x)+\theta^{\prime \prime}(x)\right) Q_{\lambda}(y) e^{i \sqrt{1+i \varepsilon}|x|} .
$$

Now it is straightforward to check that $N\left(f_{\varepsilon}\right)<\infty$. We can pass to the limit in $\varepsilon$ and we get that, setting $u_{\lambda}=\lim _{\varepsilon \rightarrow 0^{+}} u_{\lambda}^{\varepsilon}=Q_{\lambda}(y) \theta(x) e^{i|x|}$, then

$$
\Delta u_{\lambda}+n_{\lambda} u_{\lambda}=f(x, y):=\left(\operatorname{sig}(x) \theta^{\prime}(x)+\theta^{\prime \prime}(x)\right) Q_{\lambda}(y) e^{i|x|} .
$$

Also if $0<\lambda<1 / 2$ and $n_{\infty}^{\lambda}=1-\frac{\lambda^{2}}{2}$, we get

$$
n_{\lambda}-n_{\infty}^{\lambda}=\tilde{n}_{\lambda}=\lambda^{2} Q^{2}(\lambda y)
$$

Therefore

$$
r \frac{\left(\frac{\partial}{\partial r} \tilde{n}_{\lambda}\right)_{-}}{n_{\lambda}}<2 C \lambda^{2} \quad r>1,
$$

and can be made as small as wanted. On the other hand straightforward computations prove that $\frac{1}{R} \int_{B(R)}\left|u_{\lambda}\right|^{2}<\infty$,

$$
\sup _{\varepsilon} \int \mid \nabla\left(\left.e^{-i \sqrt{1+i \varepsilon}|x|} u_{\lambda}^{\varepsilon}\right|^{2} \leq c\left(1+\int\left(Q^{\prime}\right)^{2} d y\right) \leq c\right.
$$

and that there is $c_{0}>0$ independent of $\varepsilon$ such that

$$
\int\left|\nabla_{\tau} u\right|^{2} \frac{d x}{\left(1+\left(x^{2}+y^{2}\right)^{1 / 2}\right.} \geq c_{0}|\log \varepsilon| .
$$

Also notice that $\varphi=i \sqrt{1+i \varepsilon}|x|$ is not a solution of $|\nabla \varphi|^{2}=n$. 


\section{References}

[1] S. Agmon, Spectral properties of Schrödinger operators and scattering theory, Ann. Scuola Norm. Sup. Pisa, (4) 2 (1975), 151-218.

[2] S. Agmon, J. Cruz-Sampedro, I. Herbst, Generalized Fourier transform for Schrödinger operators with potentials of order zero, J. of Funct. Anal., 167 (1999), 345-369.

[3] S. Agmon, L. Hörmander, Asymptotic properties of solutions of differential equations with simple characteristics, J. Anal. Math., 30 (1976), 1-37.

[4] G. BARLes, On eikonal equations associated with Schrödinger operators with nonspherical radiation conditions, Comm. in Part. Diff. Eq., 123 (1987), 263-283.

[5] M. Ben ARTzI, A limiting absorption principle for Schrödinger operators with spherical symmetric exploding potentials, Israel J. Math., 40 (1981), 259-274.

[6] J.D. Benamou, F. Castella, T. Katsaounis, B. Perthame, High frequency limit of the Helmholtz equations, Rev. Iberoamer., 18(1)(2002), 187-209.

[7] F. Castella, B. Perthame, O. Runborg, High frequency limit of the Helmholtz equation. Source on a general manifold, Comm. in Part. Diff. Eq., 27 3-4 (2002), 607-651.

[8] D. M. Eidus, The principle of limiting absorption, Math. Sb., 57 (1962), 13-44. Amer. Math. Soc. Transl., (2) 47 (1965), 157-191.

[9] D. M. EIDus, The limiting absorption and amplitude principles for the diffraction problem with two unbounded media, Comm. Math. Phys., 107 (1986), 29-38.

[10] E. Fouassier, Morrey-Campanato estimates for Helmholtz equations with two unbounded media. arxiv.org/pdf/math.AP/0502290.

[11] I. HerBst, Spectral and scattering for Schrödinger operators with potentials independent of $|x|$, Amer. J. Math., 113 (3) (1991), 509-565.

[12] L. Hörmander, The analysis of Linear Partial Differential Operators II, GMW 257, Springer, 1990.

[13] L. Hörmander, The analysis of Linear Partial Differential Operators IV, GMW 257, Springer, 1990.

[14] T. Ikebe, Y. SAIto, Limiting absorption method and absolute continuity for the Schrödinger operator, J. Math. Kyoto Univ., 12-3 (1972), 512-542.

[15] A. Jensen, P. Perry, Commutator method and Besov space estimate for Schrödinger operators, J. Oper. Theo., 14 (1985), 181-188. 
[16] C. Kenig, G. Ponce, L. VegA, Small solutions to non-linear Schrödinger equations, Ann. Inst. H. Poincare Anal. Non Lineaire, 10 (1993), 255-288.

[17] S. T. KunodA, Scattering theory for differential operators I, operator theory, J. Math. Soc. of Japan, 25(30) (1973), 75-104.

[18] P.-L. Lions, Generalized solutions to Hamilton-Jacobi equations. Pitmann, Boston (1982).

[19] K. Mochizuki, Growth properties of solutions of second order elliptic differential equations, J. Math. Kyoto Univ., 16(2) (1976), 351-373.

[20] K. Mochizuki, J. UChiyama, Radiation conditions and spectral theory for 2-body Schrödinger operators with "oscillating" long-range potentials I, J. Math. Kyoto Univ., 18(2) (1978), 377-408.

[21] B. Perthame, L. Vega, Morrey-Campanato estimates for Helmholtz Equation, J. Funct. Anal., 164(2) (1999), 340-355.

[22] B. Perthame, L. Vega, Sommerfeld condition for a Liouville equation and concentration of trajectories, Bull. of Braz. Math. Soc., New Series 34(1) (2003), 1-15.

[23] B. Perthame, L. Vega, Energy concentration and Sommerfeld condition for Helmholtz and Liouville equations. C. R. Math. Acad. Sc. Paris, 337(9) (2003), 587-592.

[24] M. Reed, B. Simon, Analysis of Operators. Methods of Modern Mathematical Physics IV, Acad. Press, San Diego, 1978.

[25] Y. SAITO, Schrödinger operators with a nonspherical radiation condition, Pacif. J. of Math., 126(2) (1987), 331-359.

[26] Bo ZHANG, Radiation condition and limiting amplitude principle for acoustic propagators with two unbounded media, Proc. of the Royal Soc. of Edinburgh, 128 A (1998), 173-192.

[27] Bo ZHANG, On transmision problems for wave propagation in two locally perturbed half spaces, Math. Proc. Cambridge Philos. Soc., 115 (1994), 545-558.

[28] Bo Zhang, Commutator estimates, Besov spaces and scattering problems for the acoustic wave propagation in perturbed stratified fluids, Math. Proc. Cambridge Philos. Soc., 128 (2000), 177-192. 